\title{
Journal of

\section{Formation of gold nanoparticles in polymeric nanowires by low-temperature thermolysis of gold mesitylene $\dagger$}

\author{
Christoph Erk, ${ }^{a}$ Man Yan Eric Yau, ${ }^{b}$ Holger Lange, ${ }^{c}$ Christian Thomsen, ${ }^{c}$ Paul Miclea, ${ }^{d e}$ Ralf B. Wehrspohn, ${ }^{d e}$ \\ Sabine Schlecht ${ }^{* a}$ and Martin Steinhart $* f$
}

\author{
Received 26th August 2011, Accepted 19th October 2011 \\ DOI: $10.1039 / \mathrm{c} 1 \mathrm{jm} 14193 \mathrm{~g}$
}

The formation of polymer nanowires containing metal nanoparticle chains by low-temperature thermolyses of metal precursors has remained challenging. We report the block copolymer-assisted generation of locally regular chains of quasi-spherical gold nanoparticles with narrow particle diameter distribution by mild thermolysis of the non-polar gold precursor gold mesitylene inside the cylindrical nanopores of self-ordered anodic aluminium oxide (AAO). The block copolymer separates the gold mesitylene as well as the developing gold nanoparticles from the AAO pore walls so that surface nucleation and pinning of gold clusters are prevented. Growing quasi-spherical gold nanoparticles locally deform the polymer chains irreversibly adsorbed on the AAO pore walls, and the polymer chains are pushed into the space between the gold nanoparticles. The gold nanoparticles have, therefore, larger diameters and smaller specific surface than hypothetical pluglike gold entities with the same volume, the formation of which is suppressed.

\section{Introduction}

Considerable efforts have been devoted to the generation and characterization of one-dimensional nanoparticle assemblies. ${ }^{1}$ The formation of gold nanoparticle chains has turned out to be particularly challenging. Silica nanowires containing gold nanoparticle chains were obtained under the harsh conditions of vapour-liquid-solid (VLS) growth. ${ }^{2-4}$ For example, silica, ${ }^{5}$ gallium oxide, ${ }^{6}$ and magnesium oxide ${ }^{7}$ nanowires containing gold nanoparticle chains were reported to show strong wavelength-dependent and reversible photoresponse so that they may be used as wavelength-controlled optical nanoswitches. Drawbacks of VLS-based approaches are the necessity of hightemperature steps and the limited range of accessible matrix materials. One-dimensional assemblies of metal nanoparticles are also accessible under mild conditions by self-organization in

${ }^{a}$ Justus-Liebig-Universität Gießen, Institut für Anorganische und Analytische Chemie, Heinrich-Buff-Ring 58, 35392 Gießen, Germany. E-mail:Sabine.Schlecht@anorg.chemie.uni-giessen.de

${ }^{b}$ Max Planck Institute of Microstructure Physics, Weinberg 2, D-06120 Halle, Germany

'Institut für Festkörperphysik, Technische Universität Berlin, Hardenbergstraße 36, 10623 Berlin, Germany

${ }^{d}$ Institute of Physics, University of Halle-Wittenberg, Heinrich-DamerowStr. 4, 06120 Halle, Germany

${ }^{e}$ Fraunhofer Institute for Mechanics of Materials IWM, Walter-Hülse-Str. 1, 06120 Halle, Germany

${ }^{f}$ Institut für Chemie, Universität Osnabrück, Barbarastr. 7, D-46069 Osnabrück, Germany. E-mail: martin.steinhart@uni-osnabrueck.de; Fax: +49 541-9693324; Tel: +49 541-9692817

$\uparrow$ Electronic supplementary information (ESI) available. See DOI: $10.1039 / \mathrm{c} 1 \mathrm{jm} 14193 \mathrm{~g}$ the absence of shape-defining hard templates, either from preformed metal nanoparticles ${ }^{8}$ or by one-pot syntheses starting from metal precursors. ${ }^{9,10}$ However, spatial arrangement and alignment of the metal nanoparticle chains thus obtained are difficult to control.

The generation of linear gold nanoparticle assemblies by conversion of gold precursors at moderate temperatures inside the cylindrical nanopores of shape-defining hard templates, ${ }^{11}$ such as anodic aluminium oxide (AAO), has remained challenging. If gold clusters are grown on amorphous alumina, nucleation takes place at defect sites, and the clusters are released once they have reached a critical size. ${ }^{12}$ In non-epitaxial systems, even large $\mathrm{Au}$ clusters incommensurate with underlying substrates show extremely high mobility. ${ }^{13}$ As a result, conversion of polar gold precursors, such as tetrachloroaurate, in AAO is accompanied by uncontrolled diffusion and coalescence of $\mathrm{Au}$ clusters, resulting in the formation of high-aspect-ratio gold plugs. ${ }^{14}$ If solutions containing tetrachloroaurate and the block copolymer (BCP) polystyrene-block-poly(vinylpyridine) (PS- $b$ PVP), which are often used to generate two-dimensional arrays of gold nanostructures on smooth substrates, ${ }^{15}$ are infiltrated into AAO, gold clusters will mainly develop at the hydroxylterminated AAO pore walls to which tetrachloroaurate and the polar PVP blocks segregate. ${ }^{16}$

Here, we report a low-temperature bottom-up synthesis for locally ordered, linear assemblies of gold nanoparticles with narrow size distribution inside polymeric nanowires comprising thermolysis of the non-polar gold precursor gold mesitylene $\left[\mathrm{Au}_{5} \mathrm{Mes}_{5}\right] \cdot 2 \mathrm{THF}^{\mathbf{1 7 , 1 8}}$ and size-focusing particle ripening in the cylindrical nanopores of self-ordered $\mathrm{AAO}^{19}$ in the presence of 
the asymmetric $\quad$ BCP polystyrene- $b$-iso[poly(methylmethacrylate)] (PS- $b$-isoPMMA). The PS- $b$-isoPMMA keeps the gold mesitylene and the developing gold nanoparticles away from the AAO pore walls and helps prevent the formation of gold plugs.

\section{Experimental}

\section{Materials}

Gold mesitylene, a precursor yielding gold nanoparticles under anhydrous conditions, ${ }^{17}$ was synthesized following procedures reported elsewhere. ${ }^{18}$ PS- $b$-isoPMMA (polydispersity index $=$ 1.5) consisting of PS minority blocks $\left(M_{\mathrm{n}}=10000 \mathrm{~g} \mathrm{~mol}^{-1}\right)$ and isoPMMA majority blocks $\left(M_{\mathrm{n}}=46000 \mathrm{~g} \mathrm{~mol}^{-1}, 95 \%\right.$ isotactic PMMA) was supplied by Polymer Source Inc., Canada. The second differential scanning calorimetry heating run on bulk PS$b$-isoPMMA (Netzsch DCS $404 \mathrm{C}$; heating and cooling rates 10 $\mathrm{K} \min ^{-1}$ ) revealed the presence of glass transitions of isoPMMA at $\sim 61{ }^{\circ} \mathrm{C}$ and of PS at $\sim 96{ }^{\circ} \mathrm{C}$, whereas no indications of crystallization of isoPMMA were apparent in the temperature range from $25^{\circ} \mathrm{C}$ to $200^{\circ} \mathrm{C}$ (ESI, Fig. S1 $\dagger$ ). PS- $b$-isoPMMA was preferred over PS- $b$-PMMA containing atactic PMMA blocks because the low glass transition temperature of isoPMMA lies significantly below the temperature range in which gold mesitylene starts to decompose. Self-ordered AAO was prepared by the two-step anodization introduced by Masuda and Fukuda. ${ }^{19}$

\section{Preparation of nanowires}

Gold mesitylene/PS- $b$-isoPMMA nanowires were obtained by covering self-ordered AAO with a pore diameter of $35 \mathrm{~nm}$ and a pore depth of $100 \mu \mathrm{m}$ with copious amounts of gold mesitylene/ PS- $b$-isoPMMA mixtures dissolved in dichloromethane. The $\mathrm{CH}_{2} \mathrm{Cl}_{2}$ was slowly evaporated overnight through a cannula pierced through the covering of an otherwise sealed container. In the following, the nanowires are denoted $\mathrm{NW}(X / Y)-Z Z Z(X / Y=$ mass ratio gold mesitylene/PS- $b$-isoPMMA; $Z Z Z=$ highest annealing temperature applied). AAO membranes containing gold mesitylene/PS- $b$-isoPMMA nanowires were heated to 120 ${ }^{\circ} \mathrm{C}$ at a rate of $10 \mathrm{~K} \mathrm{~min}{ }^{-1}$, kept at this temperature for 20 minutes, further heated to $150{ }^{\circ} \mathrm{C}(\mathrm{NW}(X / Y)-150)$ or to $200{ }^{\circ} \mathrm{C}$ $(\mathrm{NW}(X / Y)-200)$ at a rate of $10 \mathrm{~K} \mathrm{~min}^{-1}$, and kept at the respective target temperature for 60 seconds prior to removal from the furnace. $\mathrm{NW}(X / Y)-120$ were directly removed from the furnace after heating to $120{ }^{\circ} \mathrm{C}$ for 20 minutes. The mass of the gold mesitylene/PS- $b$-isoPMMA mixtures per $\mathrm{mL}$ dichloromethane in the solutions infiltrated into the AAO membranes amounted to $42.8 \mathrm{mg}$ in the case of $\mathrm{NW}(1 / 1.0)-Z Z Z ; 39.9 \mathrm{mg}$ in the case of $\mathrm{NW}(1 / 1.1)-Z Z Z ; 38.0 \mathrm{mg}$ in the case of $\mathrm{NW}(1 / 1.3)-Z Z Z ; 35.3$ $\mathrm{mg}$ in the case of $\mathrm{NW}(1 / 1.4)-Z Z Z$; and $30.7 \mathrm{mg}$ in the case of $\mathrm{NW}$ (1/1.9)-ZZZ.

\section{Characterization of nanowires}

The Raman spectra of nanowires located inside AAO templates displayed in Fig. 5 were measured with a LabRAM HR system equipped with a HeNe-laser emitting at $632 \mathrm{~nm}$ as excitation source. The Raman spectra shown in Fig. S4 of the ESI $\uparrow$ were acquired in the same way except that the excitation wavelength was $532 \mathrm{~nm}$. All Raman spectra displayed in one figure were recorded with the same laser, the same power density and the same focal volume. The Raman spectra were normalized according to the duration of the measurements.

For UV-Vis extinction spectroscopy, the nanowires were released from the AAO membranes by etching the latter with $15 \mathrm{wt} \%$ aqueous potassium hydroxide solution. UV-Vis extinction spectra of NW(1/1.9)-120, NW(1/1.9)-150, NW(1/1.9)-200 and gold mesitylene/PS- $b$-isoPMMA nanowires without any heat treatment obtained from the same gold mesitylene/PS- $b$-isoPMMA solution were obtained from suspensions in $15 \mathrm{wt} \%$ aqueous $\mathrm{KOH}$ solution directly after etching the AAO since, under these conditions, agglomeration of the nanowires could be prevented. The UV-Vis extinction spectra were measured with a Horiba Jobin Yvon monochromator iHR 550. For excitation, a 75W Halogen lamp was used, and for signal detection a $\mathrm{Si}$ detector coupled with a lock-in amplifier was used.

For transmission electron microscopy (TEM) investigations and X-ray powder diffractometry, suspensions of released nanowires in aqueous $\mathrm{KOH}$ solutions obtained as described above were neutralized by three washing cycles including centrifugation, removal of the supernatant solution and addition of deionized water, another washing step with $\mathrm{KOH}$ solution to remove $\mathrm{Al}(\mathrm{III})$-containing precipitations and three more washing cycles with deionized water as described above. X-Ray powder diffraction patterns were obtained with a STOE STADI P transmission powder diffractometer in $\theta / 2 \theta$ geometry using $\mathrm{Cu}-\mathrm{K}_{\alpha}$ radiation. For this purpose, aqueous nanowire suspensions were mixed with a binder, dropped onto amorphous plastic foils acting as support and dried in air. For TEM investigations, a few droplets of neutral aqueous nanowire suspensions were deposited onto copper grids coated with holey carbon films. TEM investigations were performed with JEOL JEM 1010, Philips CM 12 and Philips CM 30 TEM/STEM devices equipped with $\mathrm{LaB}_{6}$ cathodes, which were operated at acceleration voltages of $100 \mathrm{kV}$ and $300 \mathrm{kV}$, respectively.

\section{Results and discussion}

Infiltration of gold mesitylene/PS- $b$-isoPMMA mixtures dissolved in dichloromethane into self-ordered AAO and subsequent evaporation of $\mathrm{CH}_{2} \mathrm{Cl}_{2}$ yielded gold mesitylene/PS- $b$ isoPMMA nanowires (Scheme 1, panel a). It is reasonable to assume that the gold mesitylene forms, as in crystals, pentameric units consisting of a gold core surrounded by a nonpolar shell of mesitylene ligands. Therefore, PS will have significantly higher affinity to gold mesitylene than isoPMMA. PMMA, on the other hand, selectively segregates to inorganic oxidic surfaces in the presence of PS, ${ }^{20,21}$ where PMMA irreversibly adsorbs, forming rigid amorphous layers even at temperatures far above its glass transition temperature. ${ }^{22}$ Thus, the PS- $b$-isoPMMA molecules inside $35 \mathrm{~nm}$ pores of the AAO will be irreversibly adsorbed on the pore walls via a rigid amorphous isoPMMA layer.

Intact pentameric gold mesitylene units appeared as dark spots and were homogeneously dispersed throughout gold mesitylene/ PS- $b$-isoPMMA nanowires before any heat treatment (Fig. 1). Besides 2,2',4,4',6,6'-hexamethylbiphenyl, as confirmed by mass spectrometry (ESI, Fig. S2 $\dagger$ ) and NMR spectroscopy (ESI, Fig. S3 $\dagger$ ), thermal decomposition of pentameric $\left[\mathrm{Au}_{5} \mathrm{Mes}_{5}\right]$ yields 


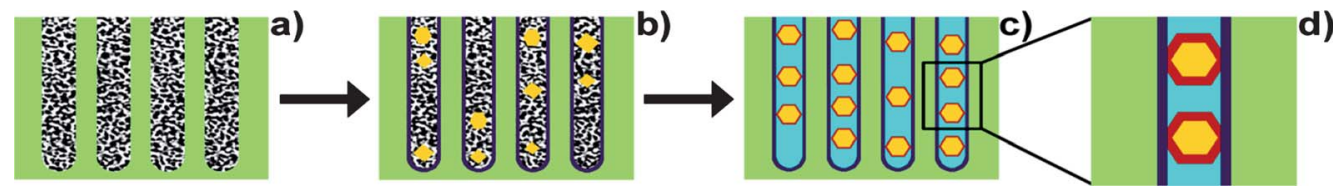

Scheme 1 Formation of gold nanoparticle chains by low-temperature thermolysis of gold mesitylene in the presence of PS- $b$-isoPMMA inside AAO. Green, AAO pore walls; white/black, gold mesitylene/PS- $b$-isoPMMA mixture; dark yellow, gold; violet, rigid isoPMMA layer at the AAO pore walls; light blue, flexible isoPMMA segments; red, PS blocks. (a) Gold mesitylene/PS- $b$-isoPMMA nanowires after evaporation of the solvent prior to thermal treatment; (b) gold/PS- $b$-isoPMMA nanowires after initial thermal treatment resulting in the development of gold nanoparticles with broad size distribution by Ostwald ripening; (c and d) gold/PS- $b$-isoPMMA nanowires after thermolysis containing faceted nanoparticles with spherical contour and narrow size distribution impinging on rigid isoPMMA layers on the AAO pore walls: (c) overview and (d) detail.

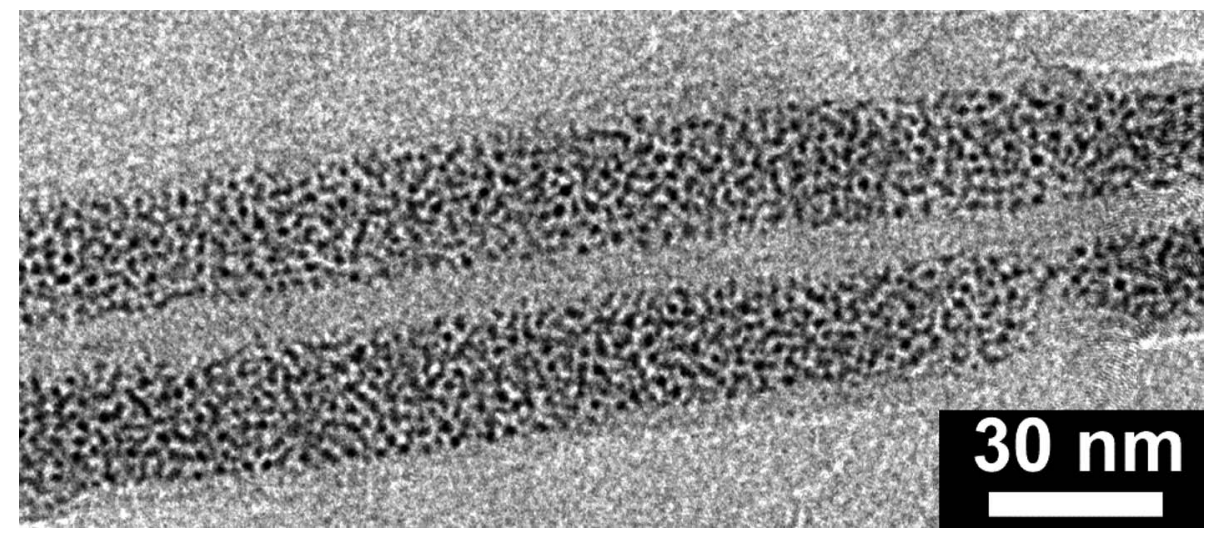

Fig. 1 TEM image of PS- $b$-isoPMMA/gold mesitylene nanowires prior to any heat treatment, which were obtained by infiltration of a 42.8 wt $\%$ solution of a gold mesitylene/PS- $b$-isoPMMA mixture (weight ratio $1: 1$ ) in $\mathrm{CH}_{2} \mathrm{Cl}_{2}$ into AAO.

small gold clusters. Ostwald ripening ${ }^{23}$ leads then to the growth of gold nanoparticles with broad size distribution (Scheme 1, panel b). Occasionally, gold nanoparticles with sizes ranging from a few nm to above $10 \mathrm{~nm}$, as in NW(1/1.3)-120 (Fig. 2a) and in the upper NW(1/1.0)-120 in Fig. 2b, had formed. Remarkably, the immediate surrounding of the gold nanoparticle seen in Fig. 2b was almost free of gold mesitylene, indicating the occurrence of particle ripening controlled by local diffusion in a limited volume. Thus, nucleation of Au clusters, which is apparently coupled with the decomposition of the gold mesitylene, occurred sporadically rather than instantaneously, as being expected for a growth regime dominated by classical Ostwald ripening. ${ }^{23}$ A precursor-free outer nanowire shell with a thickness of a few nm clearly discernable in many TEM images (see, for example, the NW(1/1.4)-120 in Fig. 2c) is most likely the signature of the rigid isoPMMA layer initially covering the AAO pore walls. Note that neither in as-infiltrated gold mesitylene/PS- $b$ isoPMMA nanowires nor in $\mathrm{NW}(X / Y)-120$ were domain structures originating from microphase separation of PS- $b$-isoPMMA apparent.

The asymmetric deformation vibration of the methyl groups at the phenyl rings of the mesitylene ligands at $1571 \mathrm{~cm}^{-1}$ was still apparent in NW(1/1.1)-120 but absent in NW(1/1.1)-150, as revealed by Raman spectroscopy (ESI, Fig. S4 $\uparrow$ ). This outcome suggests not only that the thermolysis of the gold mesitylene is largely completed in $\mathrm{NW}(X / Y)-150$ but also that the degradation product $2,2^{\prime}, 4,4^{\prime}, 6,6^{\prime}$-hexamethylbiphenyl sublimes. Indeed, we observed precipitations of colorless crystalline $2,2^{\prime}, 4,4^{\prime}, 6,6^{\prime}$-hexamethylbiphenyl at the cold end of a Schlenk tube within which gold mesitylene/PS- $b$-isoPMMA nanowires were heated ( $c f$. ESI, Fig. S2 and $\mathrm{S} 3 \dagger$ ).

Gold clusters were reported to preferentially possess icosahedral $^{24}$ or dodecahedral ${ }^{25,26}$ shapes so that their surfaces exclusively consist of $\{111\}$ facets having lower surface energy than $\{110\}$ or $\{100\}$ facets. ${ }^{27,28}$ Optimization of the $\{111\} /\{100\}$ ratio on their surfaces related to high mobility of edge atoms at elevated temperatures ${ }^{27,28}$ leads to much more pronounced and sharper facets and to a quasi-spherical shape of the growing gold nanoparticles. In $\mathrm{NW}(X / Y)-200$ conversion of gold mesitylene to gold as well as particle ripening were completed. As a result, quasi-spherical, faceted gold nanoparticles formed, as can be seen in TEM images of released NW(1/1.3)-200 (Fig. 3a) and NW (1/1.4)-200 (Fig. 3b). The gold nanoparticles were locally ordered along the nanowires on length scales of a few $100 \mathrm{~nm}$. The centerto-center distance between the gold nanoparticles seen in Fig. 3b amounted to $\sim 45 \mathrm{~nm}$. The evaluation of several TEM images revealed a narrow diameter distribution of the gold nanoparticles centering about $25 \mathrm{~nm}$. The evaluation of the full widths at half maximum of the (111), (200) and (311) reflections of facecentered cubic gold (PDF 4-0784) in X-ray powder diffraction patterns of released, randomly oriented NW(1/1.9)-200 (ESI, Fig. S5 $\dagger$ ) indicated that no preferred growth along specific crystallographic directions occurred. In accordance with the TEM results, the Scherrer method ${ }^{29}$ revealed a mean particle diameter of $\sim 25 \mathrm{~nm}$. In $\mathrm{NW}(X / Y)-150$ subjected to the same temperature profile as the $\mathrm{NW}(1 / 1.3)-200$, but heated only to $150{ }^{\circ} \mathrm{C}$, both PS- $b$-isoPMMA/gold mesitylene segments containing smaller gold nanoparticles such as the $\mathrm{NW}(X / Y)-120$ 

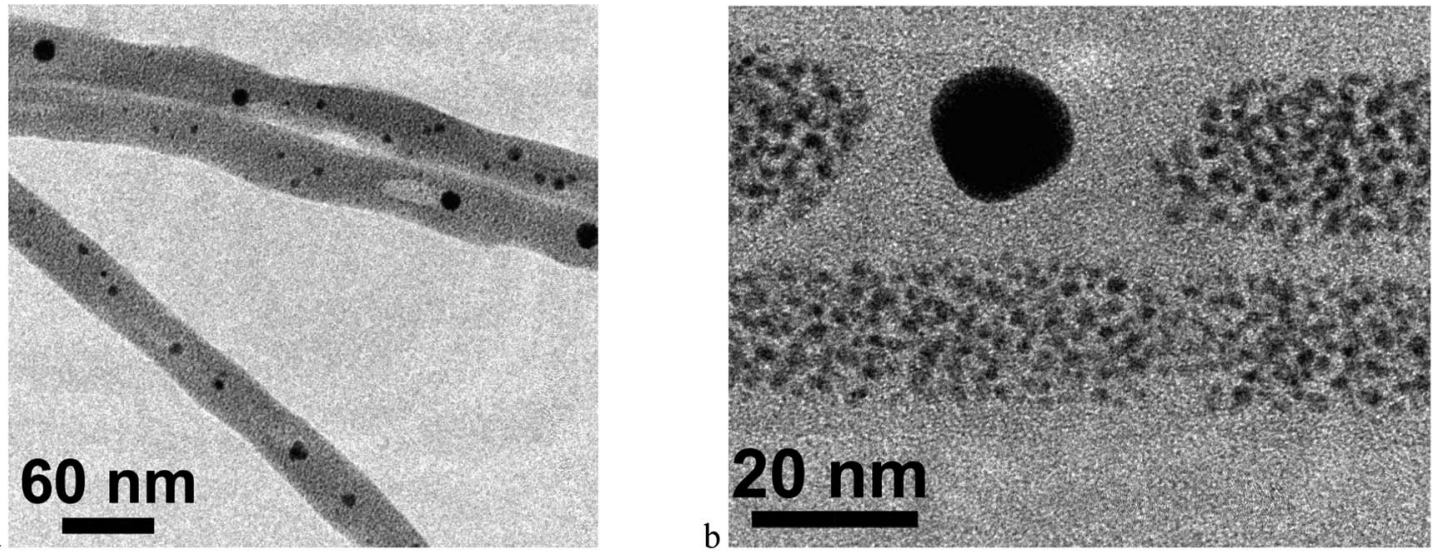

a

\section{$20 \mathrm{~nm}$}

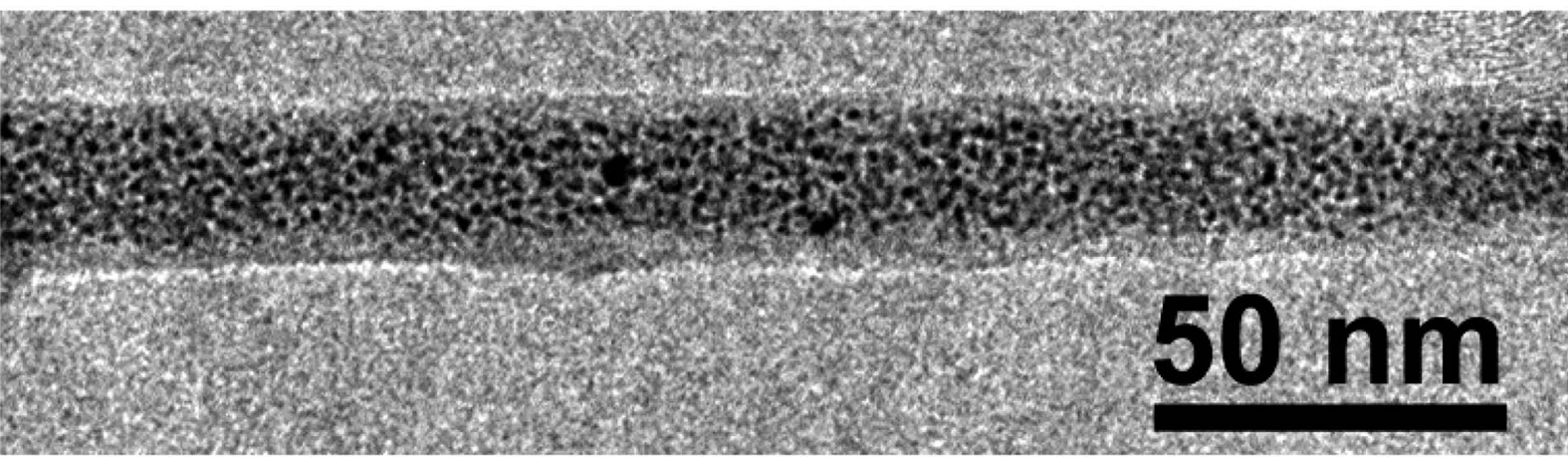

Fig. 2 Evolution of gold nanoparticles in gold mesitylene/PS-isoPMMA nanowires heated to $120^{\circ} \mathrm{C}$ : (a) NW(1/1.3)-120 with some small gold particles; (b) NW(1/1.0)-120 exhibiting one larger gold particle and a precursor-free surrounding; (c) NW(1/1.4)-120 with an outer shell of isoPMMA free of gold mesitylene.

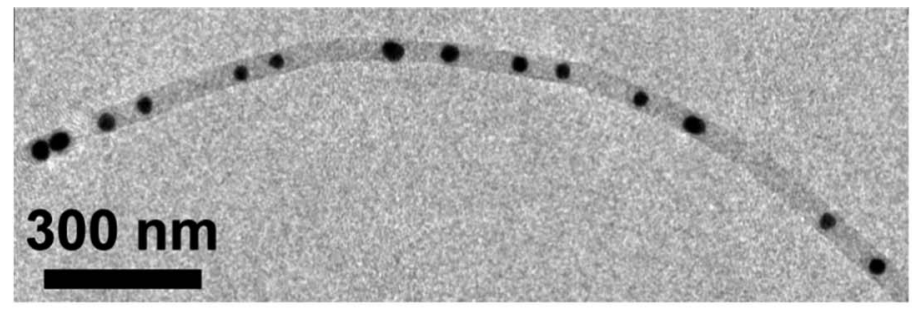

a

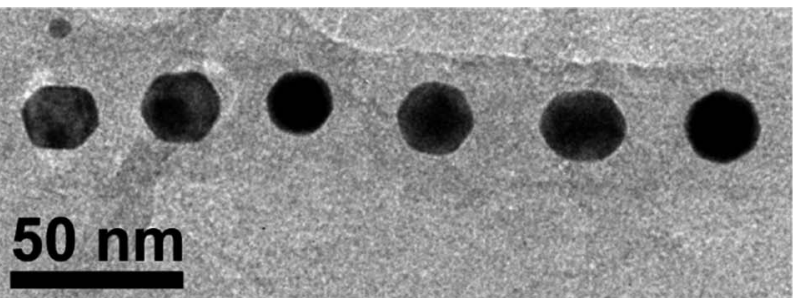

$\mathrm{b}$

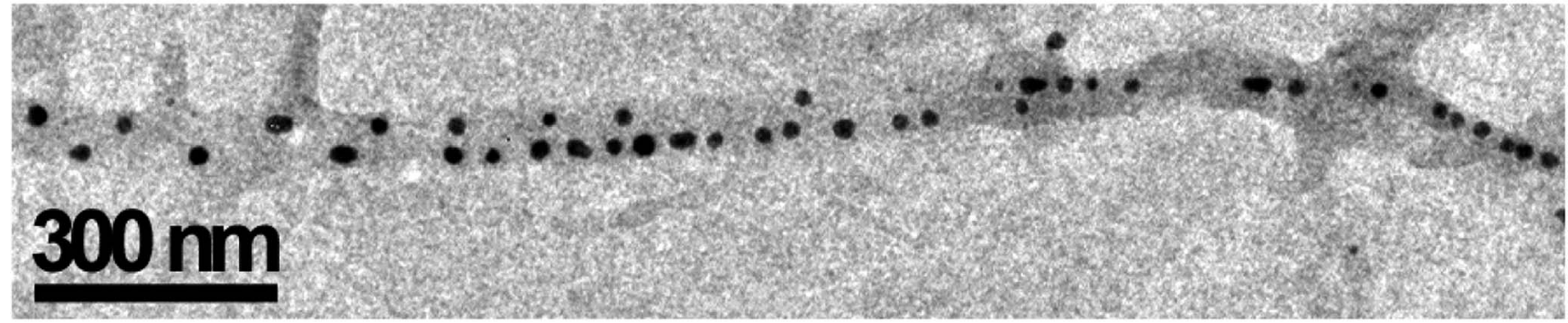

c

Fig. 3 Gold/PS- $b$-isoPMMA nanowires containing quasi-spherical gold nanoparticles with narrow size distribution: (a) large-field view of NW(1/1.3)200 and (b) detail of NW(1/1.4)-200 after thermal treatment at $200{ }^{\circ} \mathrm{C}$. Faceted gold particles about 25 nm in diameter formed; (c) NW(1/1.4)-150 showing chains of gold nanoparticles closely located to their neighbours leading to local plasmonic coupling.

shown in Fig. 2a and PS- $b$-isoPMMA/gold segments, in which the formation of gold nanoparticles was completed (Fig. 3c), occurred.
Fig. 4 shows UV-Vis extinction spectra of randomly oriented NW(1/1.9)-120, NW(1/1.9)-200 and gold mesitylene/PS- $b$-isoPMMA nanowires without any heat treatment obtained from the 


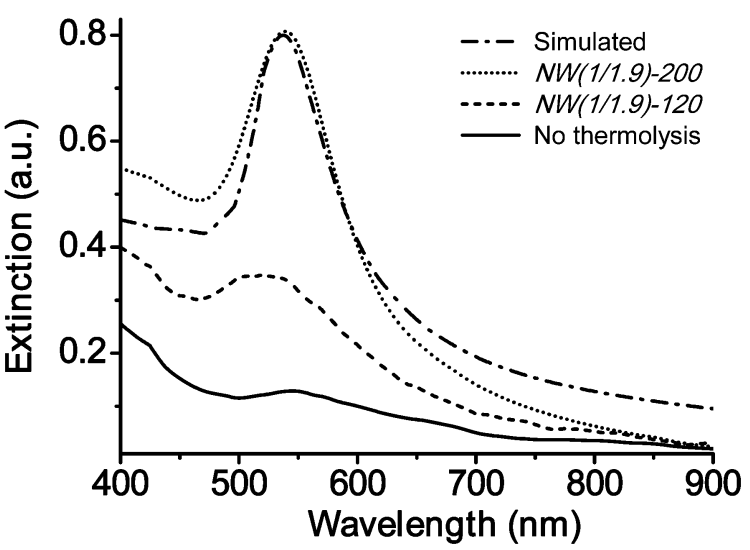

Fig. 4 UV-Vis extinction spectra of randomly oriented NW(1/1.9)-120 (dashed curve), NW(1/1.9)-200 (dotted curve) and gold mesitylene/PS- $b$ isoPMMA nanowires without any heat treatment (solid curve) obtained from the same gold mesitylene/PS- $b$-isoPMMA solution suspended in 15 wt $\%$ aqueous $\mathrm{KOH}$ solutions. Dash-dotted curve: simulated extinction spectrum assuming a log-normal nanoparticle size distribution density.

same gold mesitylene/PS- $b$-isoPMMA solution suspended in 15 $\mathrm{wt} \%$ aqueous $\mathrm{KOH}$ solution. The extinction spectrum of gold mesitylene/PS- $b$-isoPMMA nanowires prior to any heat treatment (Fig. 4, solid curve) does not show distinct plasmon absorption bands. The extinction spectrum of NW(1/1.9)-120 (Fig. 4, dashed curve) shows a weak and broad resonance at $\sim 520 \mathrm{~nm}$, indicating the beginning presence of gold nanoparticles, and that of NW(1/1.9)-200 (Fig. 4, dotted curve) shows an intense and narrow absorption band at $\sim 540 \mathrm{~nm}$ with a full width at half maximum of $75 \mathrm{~nm}$. To interpret the experimental results, we simulated extinction spectra based on the extended Mie theory ${ }^{30}$ by extending the program of $\mathrm{Xu}$ and Gustafson ${ }^{31}$ over the relevant spectral range. The extinction spectrum of sample NW(1/1.9)-200 could be reproduced by assuming an effective refractive index of 1.39 as well as a log-normal distribution density of the diameters of the gold nanoparticles with a mean particle diameter $d_{\text {mean }}$ of $15 \mathrm{~nm}$ and a standard deviation $\sigma$ of $2 \mathrm{~nm}$ :

$$
\frac{1}{\sigma d \sqrt{2 \pi}} \exp -\frac{\left(\ln d-d_{\text {mean }}\right)^{2}}{2 \sigma^{2}}
$$

In general, the simulation (Fig. 4, dash-dotted curve) suggests that Mie resonances of separated gold nanoparticles having a narrow diameter distribution are by far the dominating contribution. However, the fact that the extinction spectrum of sample NW(1/1.9)-200 was best represented by simulations based on a log-normal size distribution of the gold nanoparticles rather than based on monodisperse particle size suggests that weak contributions of coupled gold nanoparticles are also present. This outcome is in line with the presence of some gold nanoparticles located close to their nearest neighbours in Fig. 3a and c. Moreover, gold nanoparticles in two different nanowires sticking together could be close enough for plasmon coupling. Most importantly, distinct longitudinal and transversal plasmon resonances originating from elongated or rodlike gold entities were completely absent.

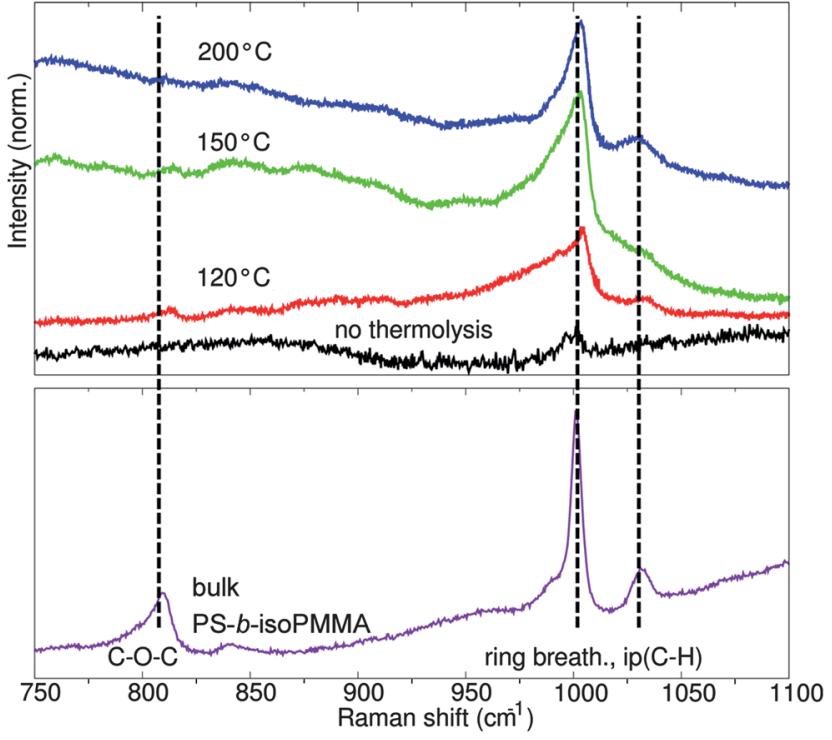

Fig. 5 Raman spectra of bulk PS- $b$-isoPMMA (violet curve), NW(1/ 1.1)-120 located in AAO (red curve), NW(1/1.1)-150 located in AAO (green curve), NW(1/1.1)-200 located in AAO (blue curve) and gold mesitylene/PS- $b$-isoPMMA nanowires without any heat treatment obtained from the same gold mesitylene/PS- $b$-isoPMMA solution located in AAO (black curve, scaled by a factor of 50).

The structure formation of $\mathrm{NW}(1 / 1.1)-Z Z Z$ in AAO membranes was monitored by Raman spectroscopy (Fig. 5). The Raman spectrum of bulk PS- $b$-isoPMMA features the $\mathrm{C}-\mathrm{O}-\mathrm{C}$ stretch of isoPMMA at $810 \mathrm{~cm}^{-1}$, the ring-breathing mode of PS at $1001 \mathrm{~cm}^{-1}$ and the in-plane $\mathrm{C}-\mathrm{H}$ vibration of PS at $1032 \mathrm{~cm}^{-1}$. The relative intensity $I_{\mathrm{PMMA}}$ of the isoPMMA C-O-C stretch at $810 \mathrm{~cm}^{-1}$ was obtained by normalizing the peak area to that of the PS ring breathing mode in the same spectrum. $I_{\text {PMMA }}$ had values of 0.35 for bulk PS- $b$-isoPMMA and 0.40 for gold mesitylene/PS- $b$-isoPMMA nanowires prior to any heat treatment, but decreased to 0.26 for sample NW(1/1.1)-120 and to 0.04 for sample NW(1/1.1)-150. In the case of sample NW(1/1.1)-200, the area of the $\mathrm{C}-\mathrm{O}-\mathrm{C}$ stretch Raman band was below the error limit so that $I_{\text {PMMA }}$ was considered zero.

Relative intensities $I_{\mathrm{PS}}$ of the PS ring-breathing mode at 1001 $\mathrm{cm}^{-1}$ were obtained by normalizing the peak areas to that in the spectrum of gold mesitylene/PS- $b$-isoPMMA nanowires prior to any heat treatment. In the spectra of NW(1/1.1)-120, NW(1/1.1)150 and $\mathrm{NW}(1 / 1.1)-200, I_{\mathrm{PS}}$ amounted to $\sim 115, \sim 250$ and $\sim 230$, respectively. The Au nanoparticles in NW(1/1.1)-120 were apparently in contact with both PS and isoPMMA. As compared to $\mathrm{NW}(1 / 1.1)-120$, for $\mathrm{NW}(1 / 1.1)-150$ a pronounced increase in $I_{\mathrm{PS}}$ is apparent. This outcome indicates the occurrence of pronounced surface-enhanced Raman scattering (SERS) ${ }^{32,33}$ in NW(1/1.1)-150, while a significantly weaker SERS effect was observed for NW(1/1.1)-120, and no SERS effect was observed for gold mesitylene/isoPMMA nanowires prior to any heat treatment. The occurrence of SERS in NW(1/1.1)-120 is related to the initial development of gold nanoparticles and the initial segregation of the PS blocks to their surfaces (Scheme 1, panel $\mathrm{b}$ and Fig. 2a). The development of the gold nanoparticles and the segregation of the PS blocks to their surfaces are already 
much more advanced in NW(1/1.1)-150. Hence, strong SERS occurs, as obvious from the pronounced increase in $I_{\mathrm{PS}}$, which is accompanied by a strong decrease in $I_{\text {PMMA }}$. This outcome is in line with previous reports according to which PS selectively wets gold in the presence of PMMA. ${ }^{34}$ The intensity enhancement of the PS ring breathing mode by factors of 230-250 upon heat treatment corresponds to an electrical field enhancement by a factor of about $\sim 4$. Since only a fraction of the PS repeat units is in tight contact with the gold nanoparticles, this value is in reasonable agreement with previously reported field enhancement factors of $10-20 .^{35,36}$

Strikingly, the $I_{\mathrm{PS}}$ value of NW(1/1.1)-200 (Scheme 1, panels c, d) is lower than that of $\mathrm{NW}(1 / 1.1)-150$. This finding can be rationalized as follows. Ostwald ripening leading to the formation of large Au nanoparticles impinging on the rigid amorphous isoPMMA layers on the pore walls at the expense of smaller $\mathrm{Au}$ nanoparticles formed by the decomposition of gold mesitylene to $\mathrm{Au}$ is completed in $\mathrm{NW}(1 / 1.1)-200$ but still incomplete in NW(1/1.1)-150. Therefore, in NW(1/1.1)-150 smaller Au nanoparticles are present, which have disappeared in NW(1/1.1)-200. These smaller $\mathrm{Au}$ nanoparticles having higher specific surfaces than their larger counterparts increase the gold surface area and, therefore, the strength of the SERS effect, in NW(1/1.1)-150.

Based on the experimental results discussed above, we rationalize the suppression of the formation of elongated pluglike gold entities as follows. In equilibrium, binary mixtures in cylindrical pores with walls selectively wetted by one component will form single cylindrical entities of the nonwetting component surrounded by the wetting component. Chains of spherical entities of the nonwetting component correspond to a nonequilibrium state characterized by larger specific interface area. ${ }^{37}$ Once radial growth of the quasi-spherical gold nanoparticles, which is strongly preferred in the absence of geometric constraints, is impeded by confinement, the specific gold surface would be further reduced by their conversion into rodlike entities growing along the AAO pores. However, no elongated gold entities were found in gold/PS- $b$-isoPMMA nanowires. This outcome can be explained as follows. Once the Au nanoparticles start to impinge on the rigid isoPMMA layer at the pore walls (Scheme 1, panels $\mathrm{c}$ and $\mathrm{d}$ ), Ostwald ripening leading to a broadening of the particle size distribution turns into a sizefocusing growth regime. While flexible yet immobilized PS- $b$ isoPMMA chain segments can sidestep into the space between the quasi-spherical gold nanoparticles, they cannot be displaced. Moreover, entropic restoring forces prevent strong chain stretching. ${ }^{38}$ Hence, the space required for growth of rodlike gold entities along the AAO pores is blocked by PS- $b$-isoPMMA chains. Thus, chains of quasi-spherical gold nanoparticles with narrow size distribution are retained. The quasi-spherical shape is stabilized by coordination of the phenyl moieties of the PS to the gold surface and because the surfaces of the gold nanoparticles predominantly consist of $\{111\}$ facets with low surface energy.

It should be noted that the structure formation mechanism outlined above implies that size and shape of the Au nanoparticles do not depend on the mass ratio gold mesitylene/PS- $b$ isoPMMA. Indeed, thorough inspections of $\mathrm{NW}(X / Y)-200$ obtained from mixtures containing gold mesitylene and PS- $b$ isoPMMA at the mass ratios considered in this work ranging from $1: 1.0$ to $1: 1.9$ by TEM revealed no significant differences in size and shape of the $\mathrm{Au}$ nanoparticles. However, it is reasonable to assume that in the case of higher contents of gold mesitylene more $\mathrm{Au}$ nanoparticles form along the nanowires. The hard confinement imposed by the AAO pore walls and the soft confinement imposed by the adsorbed PS- $b$-isoPMMA layers govern and limit the growth of the Au nanoparticles rather than the initial mass ratio of gold mesitylene and PS- $b$-isoPMMA. We also assume that for all mass ratios considered here the surfaces of the developing gold nanoparticles can be completely covered with the PS blocks. Therefore, the size of the gold nanoparticles cannot be tuned by means of the mass ratio of gold mesitylene and PS- $b$-isoPMMA.

\section{Conclusions}

Polymer nanowires containing locally regular chains of quasispherical Au nanoparticles uniform in size were obtained by lowtemperature thermolysis of gold mesitylene, a nonpolar gold precursor, in the cylindrical nanopores of rigid, shape-defining AAO hard templates. The presence of the block copolymer PS- $b$ isoPMMA appears to be crucial. While the isoPMMA blocks are irreversibly adsorbed on the AAO pore walls, the PS blocks segregate to the growing gold nanoparticles. Therefore, both the gold mesitylene and the growing gold nanoparticles are kept away from the AAO pore walls so that surface nucleation and surface pinning of gold clusters are prevented. Ostwald ripening of gold nanoparticles with small diameters turns into a confinement-induced size-focusing growth regime once the quasispherical gold nanoparticles impinge on the pore walls. This observation was explained by deformation of PS- $b$-isoPMMA molecules immobilized on the AAO pore walls through the growing gold nanoparticles so that the latter reach larger diameters and have smaller specific surfaces than hypothetic, pluglike gold cylinders with the same volume. Moreover, entropic restoring forces of the polymer chains pushed into the space between the growing gold nanoparticles prevent growth of the latter along the AAO pores. The synthetic methodology reported here might be a first step toward rational low-temperature bottom-up design of one-dimensional nanostructures having tailored plasmonic properties.

\section{Acknowledgements}

Financial support from the German Research Foundation (Priority Program 1165 "Nanowires and nanotubes"), preparation of AAO hard templates by S. Kallaus and K. Sklarek as well as help with SERS analysis by Niculina Peica are gratefully acknowledged.

\section{Notes and references}

1 Z. Y. Tang and N. A. Kotov, Adv. Mater., 2005, 17, 951-962.

2 K. Wang, S. Y. Chung and D. Kim, Appl. Phys. A: Mater. Sci. Process., 2004, 79, 895-897.

3 F. M. Kolb, A. Berger, H. Hofmeister, E. Pippel, U. Gösele and M. Zacharias, Appl. Phys. Lett., 2006, 89, 173111.

4 J. W. Tringe, G. Vanamu and S. H. Zaidi, J. Appl. Phys., 2008, 104, 094317.

5 M.-S. Hu, H.-L. Chen, C.-H. Shen, L.-S. Hong, B.-R. Huang, K.-H. Chen and L.-C. Chen, Nat. Mater., 2006, 5, 102-106. 
6 C.-H. Hsieh, L.-J. Chou, G.-R. Lin, Y. Bando and D. Golberg, Nano Lett., 2008, 8, 3081-3085.

7 W. W. Zhou, L. Sun, T. Yu, J. X. Zhang, H. Gong and H. J. Fan, Nanotechnology, 2009, 20, 455603.

8 M.-H. Wang, Y.-J. Li, Z.-X. Xie, C. Liu and E. S. Yeung, Mater. Chem. Phys., 2010, 119, 153-157.

9 B. D. Korth, P. Keng, I. Shim, S. E. Bowles, C. Tang, T. Kowalewski, K. W. Nebesny and J. Pyun, J. Am. Chem. Soc., 2006, 128, 6562-6563.

10 L. Wu, C. Shi, L. Tian and J. Zhu, J. Phys. Chem. C, 2008, 112, 319 323.

11 C. R. Martin, Science, 1994, 266, 1961-1966.

12 J. Carrey, J.-L. Maurice, F. Petroff and A. Vaurès, Phys. Rev. Lett., 2001, 86, 4600-4603.

13 P. Jensen, Rev. Mod. Phys., 1999, 71, 1695-1735.

14 P. Göring, E. Pippel, H. Hofmeister, R. B. Wehrspohn, M. Steinhart and U. Gösele, Nano Lett., 2004, 4, 1121-1125.

15 J. Chai, D. Wang, X. Fan and J. M. Buriak, Nat. Nanotechnol., 2007, 2, 500-506.

16 Y. Wang, U. Gösele and M. Steinhart, Nano Lett., 2008, 8, 35483553.

17 S. D. Bunge, T. J. Boyle and T. J. Headley, Nano Lett., 2003, 3, 901905.

18 E. M. Meyer, S. Gambarotta, C. Floriani, A. Chiesi-Villa and C. Guastini, Organometallics, 1989, 8, 1067-1079.

19 H. Masuda and K. Fukuda, Science, 1995, 268, 1466-1468.

20 D. Chen, J.-T. Chen, E. Glogowski, T. Emrick and T. P. Russell, Macromol. Rapid Commun., 2009, 30, 377-383.

21 E. Buck and J. Fuhrmann, Macromolecules, 2001, 34, 2172-2178.
22 A. Sargsyan, A. Tonoyan, S. Davtyan and C. Schick, Eur. Polym. J., 2007, 43, 3113-3127.

23 W. Ostwald, Z. Phys. Chem., 1900, 34, 495-503.

24 A. MacKay, Acta Crystallogr., 1962, 15, 916-918.

25 L. D. Marks, Surf. Sci., 1985, 150, 358-366.

26 A. S. Barnard and L. A. Curtiss, ChemPhysChem, 2006, 7, 1544-1553.

27 L. D. Marks, Rep. Prog. Phys., 1994, 57, 603-649.

28 E. Carbó-Argibay, B. Rodríguez-Gonzáles, I. Pastoriza-Santos, J. Pérez-Juste and L. M. Liz-Marzán, Nanoscale, 2010, 2, 2377-2383.

29 P. Scherrer, Nachrichten von der Gesellschaft der Wissenschaften zu Göttingen, Mathematisch-Physikalische Klasse 1918, vol. 2, pp. 98100.

30 G. Mie, Ann. Phys., 1908, 25, 377-445.

31 Y. L. Xu and B. A. S. Gustafson, J. Quant. Spectrosc. Radiat. Transfer, 2001, 70, 395-419.

32 M. Fleischmann, P. J. Hendra and A. J. McQuillan, Chem. Phys. Lett., 1974, 26, 163-166.

33 D. L. Jeanmaire and R. P. Van Duyne, J. Electroanal. Chem., 1977, 84, 1-20.

34 T. P. Russell, G. Coulon, V. R. Deline and D. C. Miller, Macromolecules, 1989, 22, 4600-4606.

35 H. X. Xu, J. Aizpurua, M. Käll and P. Apell, Phys. Rev. E: Stat. Phys., Plasmas, Fluids, Relat. Interdiscip. Top., 2000, 62, 4318-4324.

36 K. L. Kelly, E. Coronado, L. L. Zhao and G. C. Schatz, J. Phys. Chem. B, 2003, 107, 668-677.

37 A. J. Liu, D. J. Durian, E. Herbolzheimer and S. A. Safran, Phys. Rev. Lett., 1990, 65, 1897-1900.

38 P. Pincus, Macromolecules, 1976, 9, 386-388. 